PROCEEDINGS OF THE

AMERICAN MATHEMATICAL SOCIETY

Volume 137, Number 3, March 2009, Pages 891-898

S 0002-9939(08)09591-9

Article electronically published on September 24, 2008

\title{
RATIONAL HOMOTOPY OF THE POLYHEDRAL PRODUCT FUNCTOR
}

\author{
YVES FÉLIX AND DANIEL TANRÉ
}

(Communicated by Paul Goerss)

\begin{abstract}
Let $(X, *)$ be a pointed $C W$-complex, $K$ be a simplicial complex on $n$ vertices and $X^{K}$ be the associated polyhedral power. In this paper, we construct a Sullivan model of $X^{K}$ from $K$ and from a model of $X$.

Let $\mathcal{F}(K, X)$ be the homotopy fiber of the inclusion $X^{K} \rightarrow X^{n}$. Recent results of Grbić and Theriault, on one side, and of Denham and Suciu, on the other side, show the diversity of the possible homotopy types for $\mathcal{F}(K, X)$. Here, we prove that the corresponding map between Sullivan models is Golod attached, generalizing a result of J. Backelin. This property is deduced from the existence of a succession of fibrations whose fibers are suspensions.

We consider also the Lusternik-Schnirelmann category of $X^{K}$. In the case that cat $X^{n}=n$ cat $X$, we prove that cat $X^{K}=(\operatorname{cat} X)(1+\operatorname{dim} K)$.

Finally, we mention that this work is written in the case of a sequence of pairs, $\underline{X}=\left(X_{i}, A_{i}\right)_{1 \leq i \leq n}$, as in a recent work of Bahri, Bendersky, Cohen and Gitler.
\end{abstract}

The Davis-Januszkiewicz space (see 9]) is defined by the Borel construction $\mathcal{Z}_{K} \times_{T^{n}} E T^{n}$, where $\mathcal{Z}_{K}$ is the moment angle complex associated to a simplicial complex $K$ on $n$ vertices. This space is an example of the $K$ th power of a space, introduced by Buchstaber and Panov in [7] and by Strickland in [17. Many properties of this polyhedral power have been developed by Notbohm and Ray in [16] and by Grbić and Theriault in [14. We are using here a general version defined by Bahri, Bendersky, Cohen and Gitler in 5 that we recall now.

Let $K$ be a simplicial complex on $n$ vertices and $\underline{X}=\left(X_{i}, A_{i}\right)_{1 \leq i \leq n}$ be a finite sequence of pairs of CW-complexes. For each simplex $\sigma \in K$, we denote by $\underline{X}^{\sigma}$ the subspace of the product space $\prod_{i=1}^{n} X_{i}$ defined by

$$
\underline{X}^{\sigma}=\prod_{i=1}^{n} Y_{i}, \quad \text { where } Y_{i}= \begin{cases}X_{i}, & \text { if } i \in \sigma, \\ A_{i}, & \text { if } i \notin \sigma .\end{cases}
$$

The polyhedral product determined by $\underline{X}$ and $K$ is the union

$$
\underline{X}^{K}=\bigcup_{\sigma \in K} \underline{X}^{\sigma} \subset \prod_{i=1}^{n} X_{i}
$$

If we need a pointed situation, we choose a base-point $x_{i}$ in each $A_{i}$, and we point $\underline{X}^{K}$ by $\left(x_{1}, \ldots, x_{n}\right)$. When $X_{i}=X$ and $A_{i}=*$ for all $i$, we denote the polyhedral product by $X^{K}$.

Received by the editors January 22, 2008, and, in revised form, March 21, 2008.

2000 Mathematics Subject Classification. Primary 13F55, 55P62, 55U10.

(C)2008 American Mathematical Society 
When $X=B S^{1}$, the homotopy fiber of $X^{K} \rightarrow X^{n}$ is the usual moment-angle complex $\mathcal{Z}_{K}$ and is homotopy equivalent to the complement $U(K)$ of the coordinate subspaces arrangement associated to $K$ (cf. [7]):

$$
U(K)=\mathbb{C}^{n}-\bigcup_{\sigma \notin K}\left\{\left(z_{1}, \ldots, z_{n}\right) \mid z_{i_{1}}=\cdots=z_{i_{k}}=0, \text { if } \sigma=\left\{i_{1}, \ldots, i_{k}\right\}\right\} .
$$

In this paper, when $\underline{X}$ is a sequence of nilpotent spaces of finite type, we describe the rational homotopy type of $\underline{X}^{K}$ and the rational homotopy type of the homotopy fiber of the injection $\underline{X}^{K} \rightarrow \prod_{i=1}^{n} X_{i}$. Recall that a (rational) model of a connected space $X$ is a commutative differential graded $\mathbb{Q}$-algebra, $\mathcal{M}$, which is connected, of finite type and quasi-isomorphic to the minimal model of $X$. More generally, we refer to [12] for basic information on rational homotopy theory and Sullivan theory of models. Also, in different parts of this paper, we use the fact that colimits on simplicial sets give homotopy colimits on the associated polyhedral product and refer to [16] for these properties.

For any $i, 1 \leq i \leq n$, we consider a surjective model $\varphi_{i}: \mathcal{M}_{i} \rightarrow \mathcal{M}_{i}^{\prime}$ of the canonical inclusion $A_{i} \hookrightarrow X_{i}$. For each $\sigma \notin K$, denote by $I_{\sigma}$ the ideal of $\otimes_{i=1}^{n} \mathcal{M}_{i}$ defined by

$$
I_{\sigma}=E_{1} \otimes \cdots \otimes E_{n} \quad \text { with } \quad E_{i}= \begin{cases}\operatorname{ker}\left(\varphi_{i}\right), & \text { if } i \in \sigma \\ \mathcal{M}_{i}, & \text { if } i \notin \sigma .\end{cases}
$$

The ideal $I(K)=\sum_{\sigma \notin K} I_{\sigma}$ brings us a model of the polyhedral product as follows.

Theorem 1. Let $\underline{X}=\left(X_{i}, A_{i}\right)_{1 \leq i \leq n}$ be a finite sequence of pairs of nilpotent $C W$ complexes of finite type. If we choose a surjective model, $\varphi_{i}: \mathcal{M}_{i} \rightarrow \mathcal{M}_{i}^{\prime}$, of the canonical inclusion $A_{i} \hookrightarrow X_{i}$, then

(1) the quotient $\otimes_{i=1}^{n} \mathcal{M}_{i} / I(K)$ is a model of $\underline{X}^{K}$.

(2) Moreover, if $J \subset K$ is a subcomplex, then the projection

$$
p_{K, J}: \otimes_{i=1}^{n} \mathcal{M}_{i} / I(K) \rightarrow \otimes_{i=1}^{n} \mathcal{M}_{i} / I(J)
$$

is a model of the inclusion $\underline{X}^{J} \hookrightarrow \underline{X}^{K}$.

As a direct consequence, we see that $\underline{X}^{K}$ is formal if the maps $\varphi_{i}$ are formalisable (see 13 or 18 for a definition and some properties of this notion), due to the fact that the cofiber of a formalisable map is formal. In particular, we recover a result of Notbohm and Ray (see [16, Theorem 5.5]): if the space $X$ is formal, then $X^{K}$ is also formal. Note that the result of Notbohm and Ray is more general and works over an arbitrary commutative ring.

Theorem 1 is exactly the rational homotopy version of the result of Bahri, Bendersky, Cohen and Gitler concerning the cohomology of $\underline{X}^{K}$. For any field $\mathbb{F}$, the authors prove, in [5, Theorem 1.22], that there is an isomorphism of algebras $H^{*}\left(\underline{X}^{K} ; \mathbb{F}\right) \cong \otimes_{i=1}^{n} H^{*}\left(X_{i} ; \mathbb{F}\right) / I(S R)$, where $I(S R)$ is the Stanley-Reisner ideal generated by monomials $x_{i_{1}} \cdots x_{i_{k}}$ for which the $x_{i}$ are elements in $H^{*}\left(X_{i} ; \mathbb{F}\right)$ and the sequence $I=\left(i_{1}, \ldots, i_{k}\right)$ for $1 \leq i_{1} \leq \cdots \leq i_{k} \leq n$ does not correspond to a simplex of $K$.

We consider now the homotopy fiber $\mathcal{F}\left(K, B S^{1}\right)$ of $\left(B S^{1}\right)^{K} \rightarrow\left(B S^{1}\right)^{n}$. In [14, Theorems 1.2 and 1.3], Grbić and Theriault provide us with sufficient conditions on $K$ which give a fiber $\mathcal{F}\left(K, B S^{1}\right)$ with the homotopy type of a wedge of spheres. Recall that a local homomorphism $f: R \rightarrow S$ is said to be $\operatorname{Golod}$ if $\operatorname{Tor}^{R}(S, k)$ has trivial Massey products of all orders $\geq 2$; see [2] for more details. On the topological 
side of the looking glass described in [1, this notion corresponds to the fact that the homotopy fiber of $f$ has the rational homotopy type of a wedge of spheres. Therefore, in terms of rational models, the result of Grbic and Theriault means: with some hypotheses on $K$, the canonical map $\mathbb{Q}\left[x_{1}, \ldots, x_{n}\right] \rightarrow \mathbb{Q}\left[x_{1} \ldots, x_{n}\right] / I(S R)$ is Golod, with $x_{i}$ of degree 2. Jörgen Backelin has proved (see [3] and [4) that, in this case, the map $\mathbb{Q}\left[x_{1}, \ldots, x_{n}\right] \rightarrow \mathbb{Q}\left[x_{1}, \ldots, x_{n}\right] / I(S R)$ can always be decomposed in a finite sequence of surjective maps that are Golod. Such a property is called Golod attached. Here, we prove that this result is true in general: for any sequence, $\underline{X}=\left(X_{i}, A_{i}\right)_{1 \leq i \leq n}$, of nilpotent spaces of finite type, the map $\otimes_{i=1}^{n} \mathcal{M}_{i} \rightarrow \otimes_{i=1}^{n} \mathcal{M}_{i} / I(K)$ is Golod attached. In fact, this result comes from the next statement written at the level of spaces.

Theorem 2. If $\underline{X}=\left(X_{i}, A_{i}\right)_{1 \leq i \leq n}$ is a finite sequence of pairs of $C W$-complexes, then there is a sequence of fibrations,

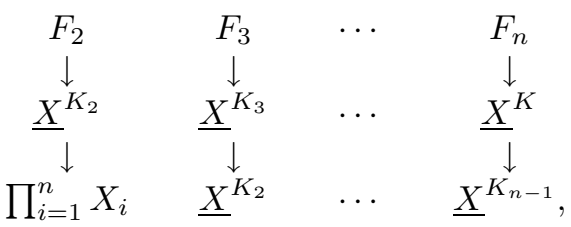

where the $F_{i}$ are suspensions and the $K_{i}$ are sub-complexes of $K$.

A precise description of the $K_{i}$ is given in the proof. From the "Golod attached" property, we deduce a nice decomposition of the rational Lie algebra of homotopy of $\mathcal{F}(K, \underline{X})$ and the fact that, generically, $\pi_{*}(\Omega(\mathcal{F}(K, X))$ contains a free Lie algebra on two generators.

Corollary 1. Suppose that the $C W$-pairs $\underline{X}=\left(X_{i}, A_{i}\right)_{1 \leq i \leq n}$ are nilpotent spaces of finite type and let $\mathcal{F}(K, \underline{X})$ be the homotopy fiber of $\underline{X}^{K} \rightarrow \prod_{i=1}^{n} X_{i}$. Then there exist an integer $N$ and a sequence of short exact sequences of graded Lie algebras where the $L_{i}$ are free graded Lie algebras defined over the rationals,

$$
\begin{aligned}
& 0 \quad \rightarrow \quad L_{n} \quad \rightarrow \quad \pi_{\geq N}(\Omega \mathcal{F}(K, \underline{X})) \otimes \mathbb{Q} \rightarrow E_{n-1} \rightarrow 0, \\
& 0 \rightarrow L_{n-1} \rightarrow \quad E_{n-1} \quad \rightarrow \quad E_{n-2} \rightarrow 0 \text {, }
\end{aligned}
$$

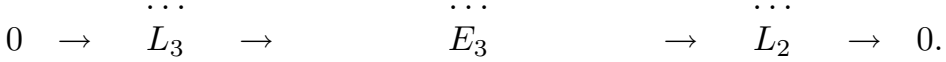

Corollary 2. We take over the notation and hypotheses of Corollary 1 and suppose also that $X_{i}=X$ and $A_{i}=*$ for $i=1, \ldots, n$. If $K \neq \Delta^{n-1}$ and if the algebra of cohomology $H^{*}(X ; \mathbb{Q})$ is not a polynomial algebra $\mathbb{Q}[\alpha]$ on a generator of even degree, then the rational Lie algebra of homotopy, $\pi_{*}(\Omega(\mathcal{F}(K, X)))$, contains a free Lie algebra on two generators.

\section{A MOdel FOR THE POLYHEDRAL PRODUCT $\underline{X}^{K}$}

Proof of Theorem 1. We work by induction on the dimension of $K$ and on the number of simplices of maximal dimension. Let $\sigma$ be a simplex of maximal dimension and suppose that $\sigma=\{1,2, \ldots, r\}$. Then we decompose $K$ in $K=K^{\prime} \cup\{1,2, \ldots, r\}$, where $K^{\prime}$ is obtained from $K$ by deleting the simplex $\sigma$.

If we have a pair of simplicial sets, $L \subset M$, we denote by $j_{M, L}: I(M) \rightarrow I(L)$ the canonical inclusion and by $p_{M, L}: \otimes_{i=1}^{n} \mathcal{M}_{i} / I(M) \rightarrow \otimes_{i=1}^{n} \mathcal{M}_{i} / I(L)$ the associated projection. 
Observe that the simplices that are not in $K$ are exactly the simplices that are not in $K^{\prime}$ and not in $\{1, \ldots, r\}$. We thus have a short exact sequence of complexes,

$$
0 \longrightarrow I(K) \stackrel{i_{1}}{\longrightarrow} I\left(K^{\prime}\right) \oplus I\left(\Delta^{r-1}\right) \stackrel{i_{2}}{\longrightarrow} I\left(\partial \Delta^{r-1}\right) \longrightarrow 0,
$$

with $i_{1}=\left(j_{K, K^{\prime}}, j_{K, \Delta^{r-1}}\right)$ and $i_{2}=j_{K^{\prime}, \partial \Delta^{r-1}}-j_{\Delta^{r-1}, \partial \Delta^{r-1}}$.

We first prove that the projections $p_{K, K^{\prime}}: \otimes_{i=1}^{n} \mathcal{M}_{i} / I(K) \rightarrow \otimes_{i=1}^{n} \mathcal{M}_{i} / I\left(K^{\prime}\right)$ and $p_{K, \Delta^{r-1}}: \otimes_{i=1}^{n} \mathcal{M}_{i} / I(K) \rightarrow \otimes_{i=1}^{n} \mathcal{M}_{i} / I\left(\Delta^{r-1}\right)$ are models of the injections $\underline{X}^{K^{\prime}} \hookrightarrow \underline{X}^{K}$ and $\underline{X}^{\Delta^{r-1}} \hookrightarrow \underline{X}^{K}$. Since

$$
I\left(\Delta^{r-1}\right)=\sum_{j=0}^{n-r-1}\left(\otimes_{i=1}^{r+j} \mathcal{M}_{i}\right) \otimes \operatorname{ker} \varphi_{j} \otimes\left(\otimes_{i=r+j+2}^{n} \mathcal{M}_{i}\right),
$$

the quotient $\otimes_{i=1}^{n} \mathcal{M}_{i} / I\left(\Delta^{r-1}\right)$ is isomorphic to $\left(\otimes_{i=1}^{r} \mathcal{M}_{i}\right) \otimes\left(\otimes_{i=r+1}^{n} \mathcal{M}_{i}^{\prime}\right)$. The quotient of $\otimes_{i=1}^{n} \mathcal{M}_{i}$ by the short exact sequence of ideals gives a new short exact sequence of complexes,

$$
\begin{aligned}
& 0 \rightarrow \otimes_{i=1}^{n} \mathcal{M}_{i} / I(K) \stackrel{p_{1}}{\longrightarrow}\left(\otimes_{i=1}^{n} \mathcal{M}_{i} / I\left(K^{\prime}\right)\right) \oplus\left(\left(\otimes_{i=1}^{r} \mathcal{M}_{i}\right) \otimes\left(\otimes_{i=r+1}^{n} \mathcal{M}_{i}^{\prime}\right)\right) \downarrow^{p_{2}} \\
&\left(\otimes_{i=1}^{r} \mathcal{M}_{i} / \otimes_{i=1}^{r} \operatorname{ker} \varphi_{i}\right) \otimes\left(\otimes_{i=r+1}^{n} \mathcal{M}_{i}^{\prime}\right) \longrightarrow 0,
\end{aligned}
$$

where $p_{1}=\left(p_{K, K^{\prime}}, p_{K, \Delta^{r-1}}\right)$ and $p_{2}=p_{K^{\prime}, \partial \Delta^{r-1}}-p_{\Delta^{r-1}, \partial \Delta^{r-1}}$.

From induction and [12, Proposition 13.5], we deduce that $\otimes_{i=1}^{n} \mathcal{M}_{i} / I(K)$ is a model for $\underline{X}^{K}, p_{K, K^{\prime}}$ a model of the inclusion $\underline{X}^{K^{\prime}} \hookrightarrow \underline{X}^{K}$ and $p_{K, \Delta^{r-1}}$ a model of the inclusion $\underline{X}^{\Delta^{r-1}} \hookrightarrow \underline{X}^{K}$.

When $J \subset K$ is a sub-complex there exists a simplex of maximal dimension, $\Delta^{r-1}$, such that the injection $J \hookrightarrow K$ factorizes via $K^{\prime}$ or $\Delta^{r-1}$, and this gives the second point of the statement by induction.

Remark. In the case $K=\partial \Delta^{n-1}, X_{i}=X$ and $A_{i}=*$ for $i=1, \ldots, n$, the space $X^{K}$ is the fat wedge of $n$ copies of $X$. By Theorem 1 , if $(\wedge Z, D)$ is a minimal model for $X$, then a model for $X^{K}$ is given by $(\wedge Z, D)^{\otimes n} /\left(\wedge^{+} Z\right)^{\otimes n}$. This is the model for the fat wedge described in [11].

\section{The homotopy fiber of the Inclusion $\underline{X}^{K} \rightarrow \prod_{i=1}^{n} X_{i}$}

There exist two kinds of results which show the diversity of the possible homotopy types of this fiber.

- In 14] Grbić and Theriault give sufficient conditions on $K$ for which the fiber of $\left(B S^{1}\right)^{K} \rightarrow\left(B S^{1}\right)^{n}$ is a wedge of spheres. This cannot be true in general; it suffices to consider the case where $K$ is the join of two simplicial complexes. More explicitly, if $K=S^{0} * S^{0}$, i.e. $K$ is the simplicial subcomplex on $\{1,2,3,4\}$ whose simplices in maximal degrees are $\{1,3\},\{1,4\}$, $\{2,3\}$ and $\{2,4\}$, then the fiber $\left(B S^{1}\right)^{K} \rightarrow\left(B S^{1}\right)^{n}$ has the homotopy type of $S^{3} \times S^{3}$.

- On the other hand this fiber is not always a formal space, as shown by Denham and Suciu in [10.

In this section, we give the proof of Theorem 2 which provides us with a decomposition of the fiber of $\underline{X}^{K} \rightarrow \prod_{i=1}^{n} X_{i}$ as a succession of fibrations and implies 
that the algebraic model of this map is Golod attached. We describe also a Sullivan model of the different spaces appearing in this decomposition.

Let $L \subseteq\{1, \ldots, n\}$ be any simplicial complex. We consider the following subsimplicial complexes:

- for $\sigma \in L, \operatorname{link}(\sigma)=\{\tau \in L \mid \tau \cup \sigma \in L$ and $\tau \cap \sigma=\emptyset\}$,

- for $\sigma \in L, \operatorname{star}(\sigma)=\{\tau \in L \mid \tau \cup \sigma \in L\}$,

- for any vertex $p, \operatorname{res}(1, \ldots, p)=L \cap\{1, \ldots, p\}$.

Proof of Theorem 2, We observe first that $K$ can be written as a pushout:

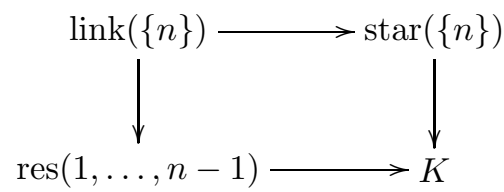

Recall from [7] that $\underline{X}^{K * L} \simeq \underline{X}^{K} \times \underline{X}^{L}$. As $\operatorname{star}(\{n\})$ is the join $\operatorname{link}(\{n\}) *\{n\}$, we have $\underline{X}^{\operatorname{star}(\{n\})}=\underline{X}^{\operatorname{link}(\{n\})} \times X_{n}$, where $\operatorname{link}(\{n\})$ is considered as a simplicial complex on $\{1, \ldots, n-1\}$. Therefore, we have a homotopy pushout

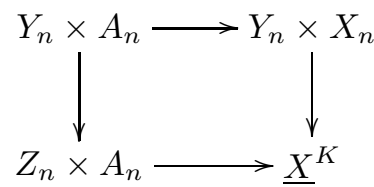

where $Y_{n}=\underline{X}^{\operatorname{link}(\{n\})}, Z_{n}=\underline{X}^{\text {res(1,..,n-1) }}$ and $A_{n} \subset X_{n}$ comes from the definition of $\underline{X}$.

First step: We set $K_{n-1}=\operatorname{res}(1, \ldots, n-1) *\{n\}$, which gives $\underline{X}^{K_{n-1}}=Z_{n} \times X_{n}$. We are looking at the homotopy fiber $F_{n}$ of $\underline{X}^{K} \rightarrow \underline{X}^{K_{n-1}}$. For that, we send each corner of the previous square onto $Z_{n} \times X_{n}$. Denote by $F_{n}^{\prime}$ the homotopy fiber of $Y_{n} \rightarrow Z_{n}$ and by $F_{n}^{\prime \prime}$ the homotopy fiber of $A_{n} \rightarrow X_{n}$. From the classical Cube Lemma (see [15]), we know that the homotopy fibers of the four previous maps onto $Z_{n} \times X_{n}$ constitute a homotopy pushout. An easy computation of three of them gives the next homotopy pushout:

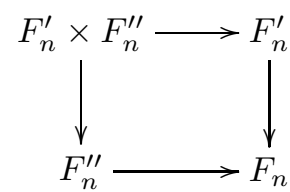

Therefore, $F_{n}$ is the join $F_{n}^{\prime} * F_{n}^{\prime \prime}$. That is a suspension.

Inductive step: Suppose that the simplicial complex $K_{p+1}$ has been constructed such that $\{p+1, \ldots, n\} \in K_{p+1}$ and $K \cap\{1, \ldots, p\}=K_{p+1} \cap\{1, \ldots, p\}$. We have a pushout:

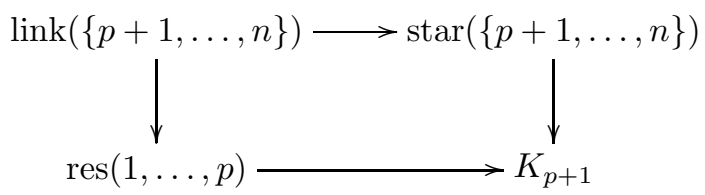


We set $K_{p}=\operatorname{res}(1, \ldots, p) *\{p+1, \ldots, n\}$, and the same argument as above gives that the fiber of $\underline{X}^{K_{p+1}} \rightarrow \underline{X}^{K_{p}}$ is a suspension. Observe that at the end of the process, $K_{1}=\Delta^{n-1}$ and $X^{\overline{K_{1}}}=\prod_{i=1}^{n} X_{i}$.

Proof of Corollary 1. The sequence of fibrations of Theorem 2 gives information on the rational homotopy of $\underline{X}^{K}$ and also on the rational homotopy of the fiber of the inclusion $\underline{X}^{K} \rightarrow \prod_{i=1}^{n} X_{i}$. Recall that, in a fibration $F \rightarrow E \rightarrow B$, the image of the connecting map $\pi_{*}(\Omega B) \otimes \mathbb{Q} \rightarrow \pi_{*-1}(\Omega F) \otimes \mathbb{Q}$ belongs to the center and that the center of a free Lie algebra on two (or more) generators is zero.

In our case, since the fibers $F_{i}$ are suspensions, we have three possibilities for their rationalisation: they are either contractible, homotopy equivalent to a sphere, or else homotopy equivalent to a wedge of at least two spheres. In the first two cases, for some $N$ we have an isomorphism $\pi_{\geq N}\left(\Omega \underline{X}^{K_{i}}\right) \otimes \mathbb{Q} \cong \pi_{\geq N}\left(\Omega \underline{X}^{K_{i+1}}\right) \otimes \mathbb{Q}$. In the third case we have a short exact sequence,

$$
0 \rightarrow L_{i} \rightarrow \pi_{*}\left(\Omega \underline{X}_{i}^{K_{i}}\right) \otimes \mathbb{Q} \rightarrow \pi_{*}\left(\Omega \underline{X}^{K_{i+1}}\right) \otimes \mathbb{Q} \rightarrow 0,
$$

where $L_{i}=\pi_{*}\left(\Omega F_{i}\right) \otimes \mathbb{Q}$ is a free graded Lie algebra. This gives the statement.

Proof of Corollary 2, Since $K \neq \Delta^{n-1}$, there exists an integer $i$ such that $K \neq$ $\{i\} *(\{1, \ldots, n\}-\{i\})$. Let $i=n$ for the sake of simplicity.

Suppose that one of the $L_{j}$ of the statement of Corollary 1 is a free Lie algebra on at least two generators. We choose $j$ maximal with this property. Thus, for some $N$, the Lie subalgebra $\left(L_{j}\right)_{\geq N}$ of the elements of degree greater than or equal to $N$ injects in $\pi_{\geq N}(\Omega(\mathcal{F}(K, X))) \otimes \mathbb{Q}$.

Finally, using the notation of the proof of Theorem 2, we observe that $F_{n}^{\prime}$ is not contractible and that the dimension of the reduced homology of $F_{n}^{\prime \prime}$ is greater than 2, from the hypothesis on the cohomology of $X$. This implies that $F_{n}$ has the rational homotopy type of a wedge of at least two spheres.

Remark. We end this section with a description of a model of the fibrations appearing in Theorem 2, For the sake of simplicity, we do it in the case $X_{i}=X$, $A_{i}=*$ for all $i$ with $X$ a nilpotent space of finite type. The extension to the case of a sequence of spaces is left to the reader.

Let $(\wedge Z, D)$ be the minimal model of $X$. It follows from Theorem 1 that a model for the inclusion $X^{K} \hookrightarrow X^{n}$ is given by the projection $(\wedge Z, D)^{\otimes n} \rightarrow$ $(\wedge Z, D)^{\otimes n} / I(K)$. Consider a relative minimal model, $(\wedge Z, D) \rightarrow(\wedge Z \otimes \wedge \bar{Z}, D)$, for the path space fibration $\Omega X \rightarrow P X \rightarrow X$. Then, a model of the homotopy fiber of the inclusion $X^{K} \rightarrow X^{n}$ is given by the tensor product

$$
(\wedge Z, D)^{\otimes n} /(I(K)) \otimes_{(\wedge Z, D)} \otimes n(\wedge Z \otimes \wedge \bar{Z}, D)^{\otimes n} .
$$

Observe that this fits with [5, Theorem 1.26], where the fiber of $X^{K} \hookrightarrow X^{n}$ is determined as the moment-angle complex associated to $(P X, \Omega X)$ and $K$.

Denote by $T$ the simplices in $\{1, \ldots, n\} \backslash K$ and decompose $T$ as $T=T_{2} \cup \ldots \cup T_{n}$, where $T_{k}$ is the subset consisting of simplices on $\{1, \ldots, k\}$ and containing $k$. Denote $I_{k}=\sum_{j \leq k} \sum_{\sigma \in T_{j}} I_{\sigma}$ and $A_{k}=(\wedge Z, D)^{\otimes k} / I_{k}$. The spaces $A_{k}$ and $A_{k+1}$ can be related as follows.

For each $\sigma \in I_{k+1} / I_{k}$, write $\sigma=\left\{i_{1}, \ldots, i_{s}\right\} \cup\{k+1\}$, with $1 \leq i_{1}<\cdots<i_{s} \leq k$, and denote by $J_{\sigma}$ the image of $B_{1} \otimes \ldots \otimes B_{k}$ in $A_{k}$, where

$$
B_{i}= \begin{cases}\wedge^{+} Z, & \text { if } i \in\left\{i_{1}, \ldots, i_{s}\right\} \\ \wedge Z, & \text { if } i \notin\left\{i_{1}, \ldots, i_{s}\right\} .\end{cases}
$$


Set $J_{k}=\sum_{\sigma \in T_{k+1}} J_{\sigma}$. Then we clearly have $A_{k+1}=A_{k} \otimes \wedge Z / J_{k} \otimes \wedge^{+} Z$. The projection $p_{k}: A_{k} \otimes(\wedge Z)^{\otimes n-k} \rightarrow A_{k+1} \otimes(\wedge Z)^{\otimes n-k-1}$ is a Sullivan model of the fibration $X^{K_{k}} \rightarrow X^{K_{k-1}}$.

Example 3. Let $X$ be a wedge of spheres with cohomology $H$ and let $K$ be a simplicial complex on $n$ vertices, of dimension $\leq 2$ and containing all the 1simplices $\{i, j\}$ for $i \neq j$. Then the homotopy fiber of the injection $X^{K} \hookrightarrow X^{n}$ has the rational homotopy type of a wedge of spheres.

For the proof, we use the minimal model of $X^{K}$. Denote first by $(H \otimes \wedge \bar{Z}, d)$ a relative minimal model of the augmentation $(H, 0) \rightarrow(\mathbb{Q}, 0)$. Then a model of the fiber is given by $A=\left(H^{\otimes n} / I(K) \otimes(\wedge \bar{Z})^{\otimes n}, D\right)$. We introduce a new gradation on $V=H \otimes \wedge \bar{Z}$ by $V=V_{0} \oplus V_{1}, V_{0}=\mathbb{Q} \otimes \wedge \bar{Z}, V_{1}=H^{+} \otimes \wedge \bar{Z}$. Note that the sequence $0 \longrightarrow \mathbb{Q} \longrightarrow V_{0} \stackrel{d}{\longrightarrow} V_{1} \longrightarrow 0$ is exact. This gradation induces a gradation on $A$ for which $A_{4}=0$ and for which the sequence $0 \longrightarrow \mathbb{Q} \longrightarrow A_{0} \stackrel{D}{\longrightarrow} A_{1} \stackrel{D}{\longrightarrow} A_{2}$ is exact. Therefore we can choose a representative for the cohomology in $A_{2} \oplus A_{3}$. Since the product of those cocycles is zero, we get a quasi-isomorphism of algebras $H(A, d) \rightarrow(A, d)$. This implies that the homotopy fiber is a wedge of spheres.

Finally, observe that, as the 2-simplices of $K$ are arbitrary, this complex is not necessarily shifted; see [14, Definition 1.1].

\section{The Lusternik-Schnirelmann CAtegory of $X^{K}$}

The (Lusternik-Schnirelmann) category of a space $X$, cat $X$, is the least integer $m$ (or $\infty$ ) such that $X$ can be covered by $m+1$ open sets each contractible in $X$. The category of a power $X^{n}$ satisfies cat $X^{n} \leq n$ cat $X$. The equality happens to be true in many situations, in particular when $X$ is a simply connected rational space [12, Theorem 30.2]; see also [6] or [8] for other examples.

Proposition 4. If $X$ is a simply connected finite type $C W$-complex such that, for any $n$, cat $X^{n}=n$. cat $X$, then the category of the polyhedral power, $X^{K}$, is equal to $(\operatorname{cat} X) \cdot(1+\operatorname{dim} K)$.

Proof. Suppose $\sigma$ is a simplex of $K$. Then $X^{\sigma} \cong X^{|\sigma|} \times\{*\}^{n-|\sigma|}$, and we have a sequence of injections $X^{\sigma} \hookrightarrow X^{K} \hookrightarrow X^{n}$. By using the canonical projection of $X^{n}$ on $X^{|\sigma|} \times\{*\}^{n-|\sigma|}$, we see that $X^{\sigma}$ is a homotopy retract of $X^{K}$. Thus we have cat $X^{K} \geq \operatorname{cat} X^{\sigma}=(\operatorname{cat} X) \cdot(1+\operatorname{dim} K)$.

On the other hand, if cat $X=m$, then $X$ is a retract of the $m$ th Ganea space, $G_{m}(X)$, which is an iterated $m$-cone. Therefore $X^{K}$ is a retract of $\left(G_{m}(X)\right)^{K}$, which is a $p$-cone with $p=m \cdot(1+\operatorname{dim} K)$. This gives the converse inequality.

\section{REFERENCES}

1. Luchezar Avramov and Stephen Halperin, Through the looking glass: a dictionary between rational homotopy theory and local algebra, Algebra, algebraic topology and their interactions (Stockholm, 1983), Lecture Notes in Math., vol. 1183, Springer, Berlin, 1986, pp. 1-27. MR 846435 (87k:55015)

2. Luchezar L. Avramov, Golod homomorphisms, Algebra, algebraic topology and their interactions (Stockholm, 1983), Lecture Notes in Math., vol. 1183, Springer, Berlin, 1986, pp. 59-78. MR.846439 (87m:13014)

3. Jörgen Backelin, Les anneaux locaux à relations monomiales ont des séries de Poincaré-Betti rationnelles, C. R. Acad. Sci. Paris Sér. I Math. 295 (1982), no. 11, 607-610. MR686351 (85a:13008) 
4. - Monomial ideal residue class rings and iterated Golod maps, Math. Scand. 53 (1983), no. 1, 16-24. MR.733934 (85h:13011)

5. A. Bahri, M. Bendersky, F. R. Cohen, and S. Gitler, The polyhedral product functor: a method of computation for moment-angle complexes, arrangements and related spaces, 2007, arXiv: 0711.4689

6. Israel Berstein, On the Lusternik-Schnirelmann category of Grassmannians, Math. Proc. Cambridge Philos. Soc. 79 (1976), no. 1, 129-134. MR0400212 (53:4047)

7. Victor M. Buchstaber and Taras E. Panov, Torus actions and their applications in topology and combinatorics, University Lecture Series, vol. 24, American Mathematical Society, Providence, RI, 2002. MR1897064 (2003e:57039)

8. Octav Cornea, Gregory Lupton, John Oprea, and Daniel Tanré, Lusternik-Schnirelmann category, Mathematical Surveys and Monographs, vol. 103, American Mathematical Society, Providence, RI, 2003. MR1990857 (2004e:55001)

9. Michael W. Davis and Tadeusz Januszkiewicz, Convex polytopes, Coxeter orbifolds and torus actions, Duke Math. J. 62 (1991), no. 2, 417-451. MR.1104531(92i:52012)

10. Graham Denham and Alexander I. Suciu, Moment-angle complexes, monomial ideals and Massey products, Pure Appl. Math. Q. 3 (2007), no. 1, 25-60. MR2330154

11. Yves Félix and Stephen Halperin, Rational LS category and its applications, Trans. Amer. Math. Soc. 273 (1982), no. 1, 1-38. MR664027 (84h:55011)

12. Yves Félix, Stephen Halperin, and Jean-Claude Thomas, Rational homotopy theory, Graduate Texts in Mathematics, vol. 205, Springer-Verlag, New York, 2001. MR1802847 (2002d:55014)

13. Yves Félix and Daniel Tanré, Formalité d'une application et suite spectrale d'Eilenberg-Moore, Algebraic topology - rational homotopy (Louvain-la-Neuve, 1986), Lecture Notes in Math., vol. 1318, Springer, Berlin, 1988, pp. 99-123. MR.952575 (89i:55007)

14. Jelena Grbić and Stephen Theriault, The homotopy type of the complement of a coordinate subspace arrangement, Topology 46 (2007), no. 4, 357-396. MR2321037

15. Michael Mather, Pull-backs in homotopy theory, Canad. J. Math. 28 (1976), 225-263. MR 0402694 (53:6510)

16. Dietrich Notbohm and Nigel Ray, On Davis-Januszkiewicz homotopy types. I. Formality and rationalisation, Algebr. Geom. Topol. 5 (2005), 31-51 (electronic). MR 2135544(2006a:55016)

17. Neil Strickland, Notes on toric spaces, preprint, 2002.

18. Micheline Vigué-Poirrier, Formalité d'une application continue, C. R. Acad. Sci. Paris Sér. A-B 289 (1979), no. 16, A809-A812. MR558804 (80k:55043)

Département de Mathématiques, Université Catholique de Louvain, 2, Chemin du Cyclotron, 1348 Louvain-La-Neuve, Belgium

E-mail address: felix@math.ucl.ac.be

Département de Mathematiques, UMR 8524, Université de Lille 1, 59655 Villeneuve D'Ascq Cedex, France

E-mail address: Daniel.Tanre@univ-lille1.fr 